\title{
PRIMER REGISTRO DE AVES FÓSILES (PELECANIFORMES: PELECANIDAE Y UN PROBABLE ODONTOPTERYGIFORMES: PELAGORNITHIDAE) PARA EL MIOCENO SUPERIOR DE COSTA RICA
}

\author{
FIRST RECORD OF FOSSIL BIRDS (PELECANIFORMES: PELECANIDAE AND \\ A PROBABLY ODONTOPTERYGIFORMES: PELAGORNITHIDAE) FROM THE \\ UPPER MIOCENE OF COSTA RICA
}

\author{
Ana L. Valerio ${ }^{1, *} \&$ César A. Laurito ${ }^{2,3}$ \\ ${ }^{1}$ Departamento de Historia Natural, Museo Nacional de Costa Rica. Apdo. \\ 749-1000, San José \\ ${ }^{2}$ INA, Instituto Nacional de Aprendizaje \\ ${ }^{3}$ Investigador Asociado-Departamento de Historia Natural, \\ Museo Nacional de Costa Rica \\ *Autor para contacto: avalerio@museocostarica.go.cr
}

(Recibido: 01/04//2013 ; aceptado: 26/11/2013)

\begin{abstract}
For the first time, remains of two fossil marine birds are recorded for Costa Rica; these fossils came out from the Upper Miocene Curré Formation. These finds are associated to shallow marine sediments and conform other marine fossil vertebrates such as cetaceans, sharks and rays.

Keywords: Birds, Pelecaniformes, Pelecanus, Odontopterygiformes, Pelagornithidae, Upper Miocene, Costa Rica.

RESUMEN: Se registran por primera vez para Costa Rica restos de dos aves marinas fósiles (Aves: Pelecaniformes y Odontopterygiformes) procedentes de la Formación Curré de edad Mioceno Superior. Estos hallazgos están asociados a sedimentos marino someros y concuerdan con otros vertebrados fósiles marinos asociados como cetáceos, tiburones y rayas.

Palabras clave: Aves, Pelecaniformes, Pelecanus, Odontopterygiformes, Pelagornithidae, Mioceno Superior, Costa Rica.
\end{abstract}




\section{INTRODUCCIÓN}

Los Pelagornithidae Fürbringer, 1888, fueron aves marinas gigantes sin representantes actuales, cuya morfología general recuerda a los pelícanos, siendo su característica más notoria el poseer grandes picos portadores de proyecciones óseas puntiagudas que semejaban dientes (Olson, 1985). Sus huesos eran excesivamente delgados por lo que su preservación es muy difícil y fragmentada, aunque recientemente Mayr \& RubilarRogers (2010), describieron un ejemplar chileno muy completo con una envergadura alar de 5.2 $\mathrm{m}$ y un peso de entre 16 y $29 \mathrm{~kg}$. Al igual que los Pelagornithidae, los Pelecanidae Rafinesque, 1815 son aves marinas con representantes actuales de amplias y largas alas, largos cuellos, grandes sacos gulares y patas cortas, con envergaduras variables entre los 1 y 1,6 $\mathrm{m}$ y pesos variables entre $\operatorname{los} 3$ y $7 \mathrm{~kg}$.

A continuación se describen dos restos óseos fósiles correspondientes a las 2 familias de Aves arriba mencionadas, los mismos proceden de la localidad fosilífera de San Gerardo de Limoncito, ubicada $14 \mathrm{~km}$ al oeste de la ciudad de San Vito en el cantón de Coto Brus, provincia de Puntarenas en las coordenadas $8^{\circ} 51^{\prime} 19.6^{\prime \prime} \mathrm{N}$ y $83^{\circ} 04^{\prime} 51.9^{\prime \prime}$ W (Fig.1). Los mismos forman parte de la Colección de Fósiles de la Sección de Geología del Museo Nacional de Costa Rica y son el objeto de estudio del presente trabajo.

\section{PALEONTOLOGÍA}

Clase AVES Linnaeus, 1758

Orden PELECANIFORMES Sharpe, 1821

Familia PELECANIDAE Rafinesque, 1815

Género PELECANUS Linnaeus, 1758

\section{Pelecanus $s p$. indet.}

Material: CFM-1689, fémur derecho sin extremo proximal (Figs. 2.2a-d).

Descripción y discusión: fragmento distal de fémur derecho en el que no se preservaron los cóndilos: lateral y mesial. Además, las fosas popliteal e intercondilar están levemente insinuadas.
El epicóndilo es rugoso y la ranura fibular profunda y elongada. El foramen nutricio es pequeño y se conserva la cresta del músculo obturador. Los cóndilos interno y externo son redondeados, la ranura patelar es una depresión redondeada, la línea anterior intermuscular no se observa.

Dimensiones: ancho mínimo del cuerpo $12,99 \mathrm{~mm}$, anchura máxima del extremo distal $17,90 \mathrm{~mm}$ y profundidad máxima del extremo distal 12,61 mm.

La epífisis distal del fémur carece de los cóndilos lateral y mesial, este hecho sugiere la posibilidad que se trataba de un espécimen juvenil; sin embargo, las dimensiones y la robustez del hueso apuntan a un ejemplar bastante grande, quizás se trataba de un individuo en transición a la adultez ya que la epífisis se encuentra soldada al resto del hueso. Otra posibilidad, es que el hueso haya sido transportado y retrabajado previo a su sedimentación definitiva y en el proceso perdió los cóndilos que suelen tener paredes óseas más delgadas y frágiles incluso en ejemplares adultos. Aparte de la no preservación de los cóndilos el fémur es indiferenciable del fémur de otras especies del género Pelecanus y difiere en forma y tamaño de otras especies de aves marinas y acuáticas con que se les comparó (ver apéndice). Otro detalle interesante de este fragmento de hueso es el ser ligeramente más robusto si se le compara con su homólogo del espécimen actual de Pelecanus occidentalis con el que se comparó (Fig. 2.1a-b), pero calza muy bien con el ejemplar de Pelecanus erythrorhyncos ilustrado por Gilbert et al. (1996) en la figura 65.

\section{Distribución paleogeográfica}

El registro fósil más antiguo del género Pelecanus, especie indeterminada data del Oligoceno Temprano (Rupeliano, de 33,0 a 28,25 Ma) del sureste de Francia (Louchart et al., 2010) y procede de sedimentos depositados en un "lagoon" dulceacuícola o ligeramente salobre, costero, de clima tropical a subtropical en el que también se registraron trogones, grullas y colibríes entre otras aves (Louchart et al., 2008). Le sigue el registro de Pelecanus tirarensis del Oligoceno Superior- Mioceno Medio de Lake Pinpa, 

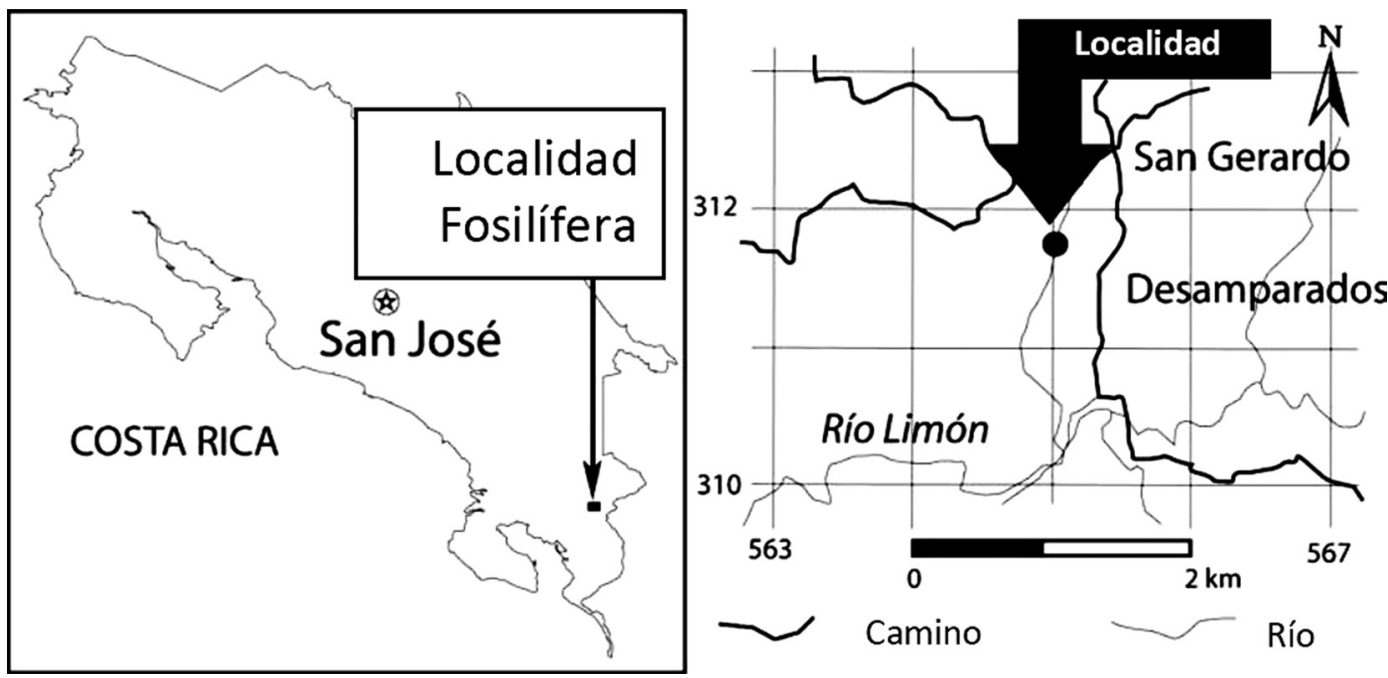

Fig.1: Mapa de ubicación de la localidad fosilífera de San Gerardo de Limoncito, cantón de Coto Brus, provincia de Puntarenas.

Australia (Miller, 1966; Rich \& van Tets, 1981); Miopelecanus gracilis Milne-Edwards, 1867 del Mioceno temprano de Francia (Cheneval, 1984), Miopelecanus intermedius (Frass, 1870) un probable sinónimo de la especie francesa y Pelecanus fraasi Lyddeker, 1891 ambas del Mioceno Medio de Lierheim, Nördlingen, Alemania (Mlískovský, 1992) y Pelecanus sivalensis Davies, 1880 del Mioceno Superior del norte de Pakistán (Brodkorb, 1963; Dyke \& Walker, 2008).

A partir del Mioceno Superior el registro fósil de Pelecanus spp. se vuelve cada vez más común y cosmopolita.

En el continente Americano, el género Pelecanus se ha registrado en el Plioceno de Oregón (Miller, 1944), Plioceno Inferior de Idaho (Wetmore, 1933; Brodkorb, 1963), La Florida y North Carolina (Olson, 1999), BlancanoIrvingtoniano del sur de California (Cassiliano, 1999), Pleistoceno de Oklahoma (Mengel, 1952), Pleistoceno tardío de Kansas (Stettenheim, 1958), todos en Estados Unidos. También, ha sido registrado en el Pleistoceno tardío del noreste del Estado de Sonora, México (Mead et al., 2006; Steadman \& Mead, 2010).

A diferencia de los relativamente abundantes registros fósiles de América del Norte, los registros de la familia Pelecanidae en América del Sur son muy raros y se limitan al hallazgo de un
Pelecanidae indeterminado en la Formación Pisco de edad Mioceno Medio-Plioceno Superior (15$2 \mathrm{Ma}$ ) (Stucchi \& Urbina, 2005) y al registro de Pelecanus sp. procedente de la localidad paleoindia de Quebrada Tacahuay del Pleistoceno tardío cuspidal (deFrance \& Urime, 2004), ambas de Perú.

Por otra parte, no existe ningún registro fósil de la familia Pelecanidae o del género Pelecanus para el Caribe a excepción del presente hallazgo.

OrdenODONTOPTERYGIFORMESHoward, 1957 Suborden ODONTOPTERYGIA Spulski, 1910 Familia PELAGORNITHIDAE Fürbringer, 1888

La familia Pelagornithidae ha sido tradicionalmente emparentada de manera muy cercana con los Pelecaniformes y en menor grado con los Procellariiformes (Olson, 1985; Warheit, 2001). Sin embargo, Smith (2010) de acuerdo a recientes análisis filogenéticos, concluye que los Pelagornithidae no pertenecen a las Neoaves; Bourdon (2005) por su parte, concluye que muy probablemente los Pelagornithidae pertenezcan al Orden Odontopterygiformes que ella considera es monofilético, además de ser el grupo hermano de los Anseriformes e incluye a ambos dentro del clado Odontoansere. Sin embargo, Mayr (2011), con base en un análisis cladístico ampliado, llega 
a la conclusión de que los Pelagornithidae junto con los Sylviornithidae y Dromornithidae, son el grupo hermano del clado Galloansere, es decir, son más cercanos a los Galliformes que a los Anseriformes, de allí que su ubicación taxonómica aún no está del todo clara.

Gen. et sp. indet.

Material: CFM-1086, fragmento proximal de ulna izquierda (Figs. 2.4a-c).

Descripción y discusión: fragmento proximal de ulna con la punta del olecranon y la superficie del cotylo dorsalis rotos. El cotylo ventralis es muy amplio y sub-circular en tanto el cotylo dorsalis está roto y su contorno real es difícil de determinar.

Dimensiones: la diagonal mayor del extremo caudal del olecranon al borde craneal del cotylo dorsalis mide $35,39 \mathrm{~mm}$ (rota) y la anchura máxima del extremo proximal mide $28,46 \mathrm{~mm}$.

El fragmento proximal de ulna es notablemente grande, casi duplica en área al extremo proximal de una ulna de Jabiru nycteria actual (Fig. 2.3) cuya envergadura alar alcanza los $2,5 \mathrm{~m}$. Ello nos permite deducir que el ave a la que perteneció la ulna fósil fue un individuo notablemente grande. Al comparar la forma y tamaño de la ulna, ésta es muy similar a la del Pelagornithidae ilustrada por Goedert (1989) en la figura $2.3 \mathrm{y}$ en general cae en el rango menor de las pocas ulnas de Pelagornithidae ilustradas en la literatura paleontológica; p.e. ver el ejemplar ilustrado por Chávez et al. (2007) en la figura 4d con dimensiones similares y en contraposición los ejemplar ilustrado por Mayr \& Zvonov (2012) en la figura 3H con tamaño que duplica el ejemplar del presente artículo.

\section{Distribución paleogeográfica}

Los Pelagornithidae han sido hallados en todos los continentes, a excepción de Australia aunque si se han registrado en Nueva Zelanda, en sedimentos que datan del Paleoceno tardío hasta el Plioceno tardío (Olson, 1985; Bourdon et al., 2010; Mayr, 2011).

En Norteamérica los registros de Pelagornithidae inician en el Eoceno tardío de Oregón (Goedert, 1989), Oligoceno tardío- Mioceno Inferior de las Formaciones Hawthorne y Ashley en South Carolina, Mioceno Medio de la Formación Calvert en Maryland y Virginia, Plioceno Inferior de Lee Creek Mine en North Carolina, todos en la costa este de Estados Unidos (Olson, 1985).

En la costa oeste de Norteamérica se han registrado en el Mioceno temprano de la Columbia Británica en Canadá (Wetmore, 1928); Mioceno Medio de Oregón y Mioceno Superior de California en Estados Unidos (Olson, 1985). En México, la Familia Pelagornithidae ha sido registrada en el Eoceno Medio de la Formación Tepetape, en Baja California Sur (González-Barba et al., 2002).

En América del Sur los Pelagornithidae han sido registrados en Perú en la Formación Pisco del Mioceno Medio-Plioceno temprano (Muizon \& DeVries, 1985) donde Chávez et al. (2007) describen Pelagornis sp.; en Chile en la Formación Bahía Inglesa del Mioceno MedioPlioceno temprano, Walsh \& Hume (2001) registran Pelagornithidae indet., Chávez et al. (2007) describen un Pelagornithidae indet. cf. Pelagornis y Pelagornis sp., por su parte Mayr \& Rubilar-Rogers (2010) describen la especie nueva Pelagornis chilensis. En Venezuela, Rincón \& Stucchi (2003) dan a conocer un Pelagornithidae indet. procedente de la Formación Capadare del Mioceno Medio del Estado de Falcón.

Aparte de los hallazgos suramericanos, cabe destacar el registro de un Pelagornithidae indet. en la Antártida, procedente de la Formación La Meseta del Eoceno-Oligoceno temprano de la isla Vicecomodoro Marambio (Tonni, 1980; Tonni \& Tambussi, 1985).

\section{Paleoecología}

Los fósiles de aves marinas son precisos indicadores paleoambientales y sus restos normalmente son hallados en sedimentos marinos 


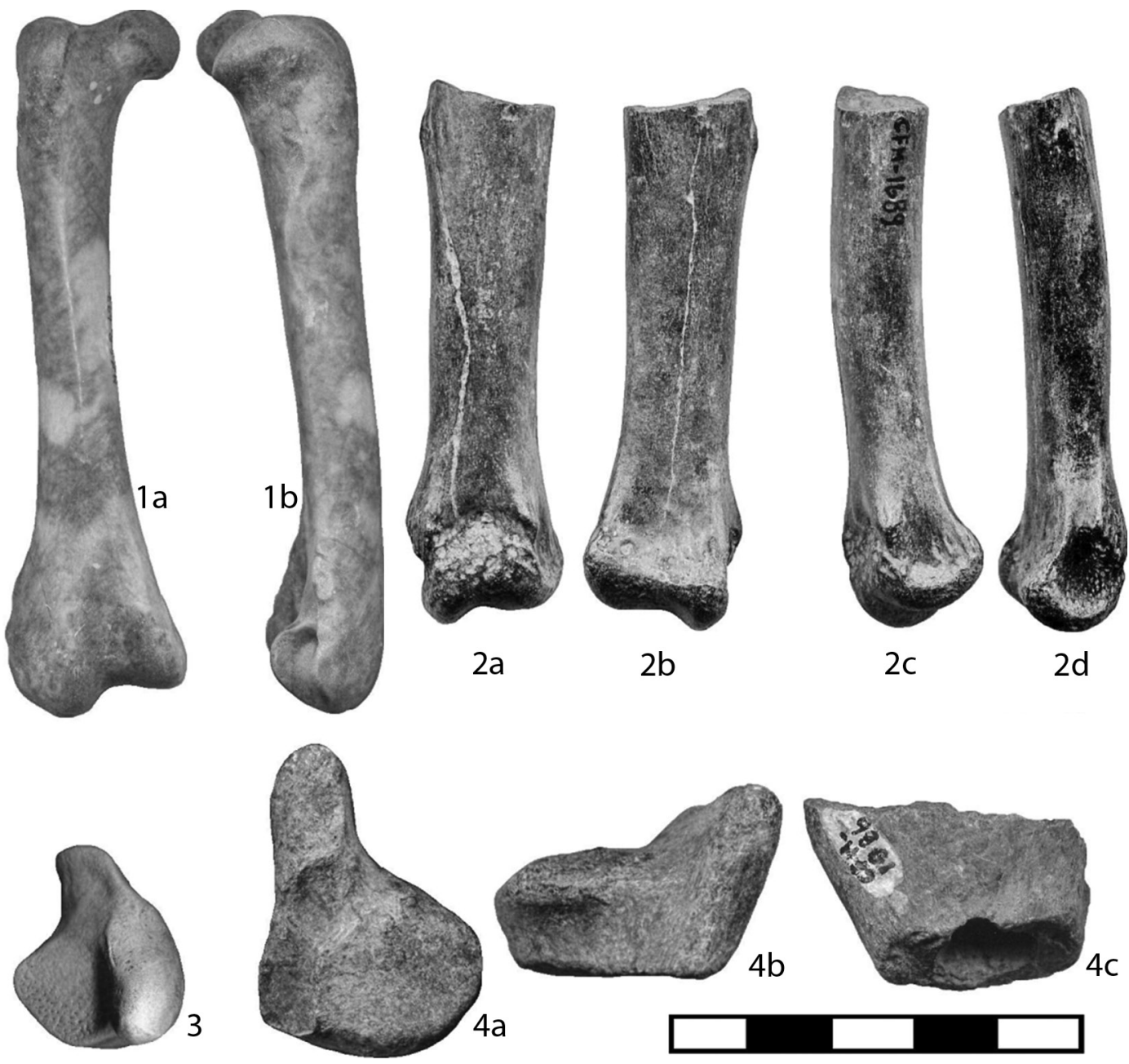

Fig- 2: 1. Fémur de Pelecanus occidentalis, a. en vista anterior, b. en vista lateral; 2. Fémur fósil de Pelecanus sp. procedente de la Fm. Curré,en vistas: a. anterior, b. posterior, c. lateral externa, d. lateral. 3. Ulna derecha de Jabiru nycteria en vista proximal y 4. Ulna izquierda de Pelagornithidae gen. et sp. indet. en vistas: a. proximal, b. palmar, c. anconal. Escala visual $5 \mathrm{~cm}$.

(Warheit, 1992), en el presente caso dicha condición es validada tanto por el contexto sedimentológico sublitoral, cuyo ambiente de depositación ha sido interpretado como el de un humedal tropical tipo estuario costero, como por el registro paleovertebradológico de cetáceos odontocetos, tortugas, cocodrilos, tiburones y rayas (Valerio, 2010; Valerio \& Laurito, 2012; Laurito \& Valerio, 2008; Laurito et al., 2005).

\section{Edad}

La edad asignada a la presente asociación de Pelecanus sp. y Pelagornithidae indeterminado es Mioceno Superior, Hemphilliano temprano (Hh1-Hh2) con base en la fauna fósil de caballos con la que se encontró asociado, a saber las especies Dinohippus mexicanus (Lance, 1950), Protohippus gidleyi Hulbert, 1988 y 
Calippus hondurensis (Olson \& McGrew, 1941) (Valerio, 2010; Laurito \& Valerio, 2010) y los Xenarthra, Pampatheriidae, Scirrohterium sp. y el Megalonychidae, Pliometanastes cf. protistus (Laurito \& Valerio, 2012a, 2012b).

\section{REFERENCIAS BIBLIOGRÁFICAS}

BOURDON, E., 2005: Osteological evidence for sister group relationship between pseudotoothed birds (Aves: Odontopterygiformes) and waterfowls (Anseriformes).Naturwissenschaften, 92: 586-591.

BOURDON, E., AMAGHZAZ, M. \& BOUYA, B., 2010: Pseudotoothed birds (Aves, Odontopterygiformes) from the early Tertiary of Morocco.- Amer. Mus. Novitates, 3704: 1-71.

BRODKORB, P., 1963: Catalogue of fossil birds. Part.1 (Archaeopterygiformes through Ardeiformes).- Bull. Florida State Mus. 7 179-293.

CASSILIANO, M.L., 1999: Biostratigraphy of Blancan and Irvingtonian Mammals in the Fish Creek-Vallecito Creek Section, Southern California, and a Review of the Blancan-Irvingtonian Boundary.- J. Vertebrate Paleontol. 19(1): 169-186.

CHÁVEZ, M., STUCCHI, M. \& URBINA, M., 2007: El registro de Pelagornithidae (Aves: Pelecaniformes) y la avifauna neógena del Pacífico sudeste.- Bull. Inst- Français d'Études Andines, 36(2): 175-197.

CHENEVAL, J., 1984: Les oiseaux aquatiques (Gaviiformes à Ansériformes) du gisement aquitanien de Saint-Gérand-le Puy (Allier, France): revision systématique.Palaeovertebrata, 14: 33-115.
DEFRANCE, S.D. \& URIME, A., 2004: Quebrada Tacahuay: un sitio marítimo del Pleistoceno tardío en la costa sur del Perú.- Chúngara: Rev. Antropol. Chilena, 36(2): 257-278.

DE MUIZON, C. \& DEVRIES, T.J., 1985: Geology and paleontology of late Cenozoic marine deposits in the Sacaco area (Peru).Geologische Rundschau, 74: 547-563.

DYKE, G. \& WALKER, C., 2008: New records of fossil "waterbirds" from the Miocene of Kenya.- Amer. Mus. Novitates, 3610: 1-10.

GILBERT, B.M., MARTIN, L.D. \& SAVAGE, H.G., 1996: Avian Osteology.- 252 págs. Missouri Archaeol. Soc., Missouri.

GOEDERT, J.L., 1989: Giant Late Eocene marine birds (Pelecaniformes: Pelagornithidae) from Northwestern Oregon.- J. Paleontol. 63(6): 939-944.

GONZÁLEZ-BARBA, G., SCHWENNICKE, T., GOEDERT, J.L. \& BARNES, L.G., 2002: Earliest Pacific basin record of the Pelagornithidae (Aves: Pelecaniformes).J. Vertebrate Paleontol. 22(3): 722-725.

LAURITO, C. \& VALERIO, A., 2008: Ictiofauna de la localidad de San Gerardo de Limoncito, Formación Curré, Mioceno Superior, cantón de Coto Brus, provincia de Puntarenas, Costa Rica.- Rev. Geol. Amér. Central, 39: 65-85.

LAURITO, C. \& VALERIO, A., 2010: Los caballos fósiles de la Formación Curré, cantón de Coto Brus, Costa Rica.- 131 págs. Mus. Nac. Costa Rica, San José.

LAURITO, C. \& VALERIO, A., 2012a: Paleobiogeografía del arribo de mamíferos suramericanos al sur de América Central 
previo al gran intercambio biótico americano: Un vistazo al GABI en América Central.- Rev. Geol. Amér. Central, 46: 123-144.

LAURITO, C. \& VALERIO, A., 2012b: Primer registro fósil de Pliometanastes sp. (Mammalia, Xenarthra, Megalonychidae) para el Mioceno Superior de Costa Rica, América Central. Una nueva pista en la comprensión del Pre-GABI.- Rev. Geol. Amér. Central, 47: 95-108.

LAURITO, C., VALERIO, A., GÓMEZ, L., MEAD, J., PÉREZ, E. \& PÉREZ, L., 2005:

A Trionychidae (Reptilia: Testudines, Cryptodira) from the Pliocene of Costa Rica, Southern Central America.- Rev. Geol. Amér. Central, 32: 7-11.

LOUCHART, A., TOURMENT, N., CARRIER, J., ROUX, T. \& MOURER-CHAUVIRÉ, C., 2008: Hummingbird with modern feathering: an exceptionally well-preserved Oligocene fossil from southern France.Naturwissenschaften, 95: 171-175.

LOUCHART, A., TOURMENT, N. \& CARRIER, J., 2010: The earliest known pelican reveals 30 million years of evolutionary stasis in beak morphology.- J. Ornithology, 152: $15-20$.

MAYR, G., 2011: Cenozoic mystery birds - on the phylogenetic affinities of bony-toothed birds (Pelagornithidae).- Zoologica Scripta, 40(5): 448-467.

MAYR, G. \& RUBILAR-ROGERS, D., 2010: Osteology of a new giant bony- toothed bird from the Miocene of Chile, with a revision of the taxonomy of Neogene Pelagornithidae.- J. Vertebrate Paleontol. 30: 1313-1330.
MAYR, G. \& ZVONOV, E., 2012: A New Genus and Species of Pelagornithidae with WellPreserved Pseudodentition and Further Avian Remains from the Middle Eocene of the Ukraine.- J. Vertebrate Paleontol. 32(4): 914-925.

MEAD, J.I., BAEZ, A., SWIFT, S.L., CARPENTER, M.C., HOLLENSHEAD, M., CZAPLEWSKI, N.J., STEADMAN, D.W., BRIGHT, J. \& ARROYO-CABRALES, J., 2006: Tropical marsh and savanna of the late Pleistocene in northeastern Sonora, Mexico.Southwest. Nat., 51: 226-239.

MENGEL, R., 1952: White pelican from the Pleistocene of Oklahoma.- The Auk, 69(1): 81-82.

MILLER, A.H., 1966: The Fossil Pelicans of Australia.- Mem. of the Queensland Mus. 14: 181-190.

MILLER, L., 1944: Some Pliocene Birds from Oregon and Idaho.- The Condor, 46(1): 25-32.

MLÍKOVSKÝ, J., 1992: The present state of knowledge of the Tertiary birds of Central Europe.- Nat. Hist. Mus. Los Angeles County, Sci. Ser. 36: 433-458.

OLSON, S.L., 1985: The fossil record of the birds.- En: FARNER, D.S., KING, J.R. \& PARKES, K.C. (eds): Avian Biology.Academic Press. 8: 79-238.

OLSON, S.L., 1999: A new species of pelican (Aves: Pelecanidae) from the Lower Pliocene of North Carolina and Florida.Proc. of the Biol. Soc. of Washington, 112(3): 503-509.

RICH, P.V.\& VANTETS, J., 1981: The fossil pelicans of Australia.- Records of the South Australian Museum (Adelaide), 18(12): 235-264. 
RINCÓN, A. \& STUCCHI, M., 2003: Primer registro de la Familia Pelagornithidae (Aves: Pelecaniformes) para Venezuela.- Bol. Soc. Venezolana de Espeleología, 37: 27-30.

SMITH, N., 2010: Phylogenetic analysis of Pelecaniformes (Aves) based on osteological data: implications for waterbird phylogeny and fossil calibration studies.- Plos one, 5(10): 1-36.

STEADMAN, D.W. \& MEAD, J.I., 2010: A Late Pleistocene Bird Community at the Northern Edge of the Tropics in Sonora, Mexico.- The American Midland Naturalist, 163(2): 423-441.

STETTENHEIM, P., 1958: Bird Fossils from the Late Pleistocene of Kansas.- The Wilson Bull. 70(2): 197-199.

STUCCHI, M. \& URBINA, M., 2005: Las Aves fósiles del Terciario peruano.- Actas del Sexto Congreso Nacional de Ornitología, Lambayeque: 134

TONNI, E P., 1980: Un pseudodontornitido (Pelecaniformes, Odontopterygia) de gran tamaño, del Terciario temprano de Antártida.- Ameghiniana, 17(3): 273-276.

TONNI, E.P. \& TAMBUSSI, C.P., 1985: Nuevos restos de Odontopterygia (Aves: Pelecaniformes) del Terciario temprano de Antártida.- Ameghiniana, 21(2-4): 121124.

VALERIO, A.L., 2010: Paleontología, bioestratigrafía y paleoecología de los caballos fósiles de la Formación Curré en el cantón de Coto Brus, Costa Rica (análisis basado en material dental).- 353 págs. Univ. de Costa Rica, San José [Tesis Lic.].
VALERIO, A.L. \& LAURITO, C.A., 2012: Cetáceos fósiles (Mammalia, Odontoceti, Eurhinodelphinoidea, Inioidea, Physeterioidea) de la Formación Curré, Mioceno Superior (Hemphilliano temprano tardío) de Costa Rica.- Rev. Geol. Amér. Central, 46: 151-160.

WARHEIT, K.I., 1992: A review of the fossil seabirds from the Tertiary of the North Pacific: plate tectonics, paleoceanography, and faunal change.- Paleobiology, 18(4): 401-424.

WARHEIT, K.I., 2001: The seabird fossil record and the role of paleontology in understanding seabird community structure.- En: SCHREIBER, E.A. \& BURGER, J. (eds): Biology of marine birds.- CRC Marine Biology Series, 17-56.

WETMORE, A., 1928: The systematic position of the fossil bird Cyphornis magnus.Canadian Department of Mines Geol. Surv. Bull. 49: 1-4.

WETMORE, A., 1933: Pliocene bird remains from Idaho.- Smithsonian Misc. Coll. 87(20): 1-12.

\section{APÉNDICE}

Ejemplares de esqueletos consultados, los cuales pertenecen a la Colección de Ornitología de la Sección de Zoología, del Museo Nacional de Costa Rica:

MNCR 028 Dendrocygna autunnalis

MNCR 029 Jabiru nycteria

MNCR 235 Sula sula

MNCR 270 Fregata magnificens

MNCR 296 Anhinga anhinga

MNCR 307 Ajaia ajaja

MNCR 697 Pelecanus occidentalis 